\title{
Hydro Electricity generation using waste water from Textile Industries through unified ETP
}

\author{
Akil Ahammed ${ }^{1}$, Tawheed Kibria ${ }^{2}$ \\ Institute of Renewable Energy Technology University of Dhaka Bangladesh \\ Institute of Renewable Energy Technology University of Dhaka Bangladesh
}

\begin{abstract}
In this project, a cutting-edge idea is shown which demonstrates the way to generate electricity using wastage water from textile industries using unified Effluent Treatment Plant (ETP). Discharged water path is from industries to river. This idea is not only a renewable project, but also saves the environment from toxic textile chemicals and unified ETP will save a huge amount of expense. As the discharged water is of low head, this problem is solved by massive discharge rate as textile industries are using huge amount of water. Necessary graphs, diagrams, charts, power calculations, efficiency etc. are shown in this paper to represent the aspect of this project.
\end{abstract}

\section{Introduction}

Hydroelectricity is one of the most renowned and well-established renewable energy technologies. It's not only a low cost source of energy but also environment friendly. Textile industries are the backbone of economy of Bangladesh. However, those are the most resource and energy consuming and environment polluting industry. Textile industries use a huge amount of water and the waste water from these industries is toxic. For this reason, these industries use ETP (effluent treatment plant), but running an ETP is costly. So for one or two shift/day, the ETP plant is kept inactive which causes severe water pollution. So a process may be introduced to save the environment, reduce the cost of the industries and can generate pollution free energy (here, hydro-electricity) before the waste water is dumped in the river.

\section{Site Location \& Resource}

Here, Gazipur area is selected and the resource water path is from the industries in that area to Turag River. The resource for this micro-hydro plant system is huge amount of waste water from textile industries. There are approx. 1700 washing and dyeing factories consuming $435000(\mathbf{1 0 \%})$ tons of water daily. The amount of exhaust water is 5-8\% reduced to consumption. As the water head (from surface to river water bed) is relatively low (15-20 feet), the high discharge rate of water will be the appropriate 'fuel'.

\section{Schematic:}

Here is a schematic shown below indicating the path of water from industries to river via ETP, reservoir and several power generating components-

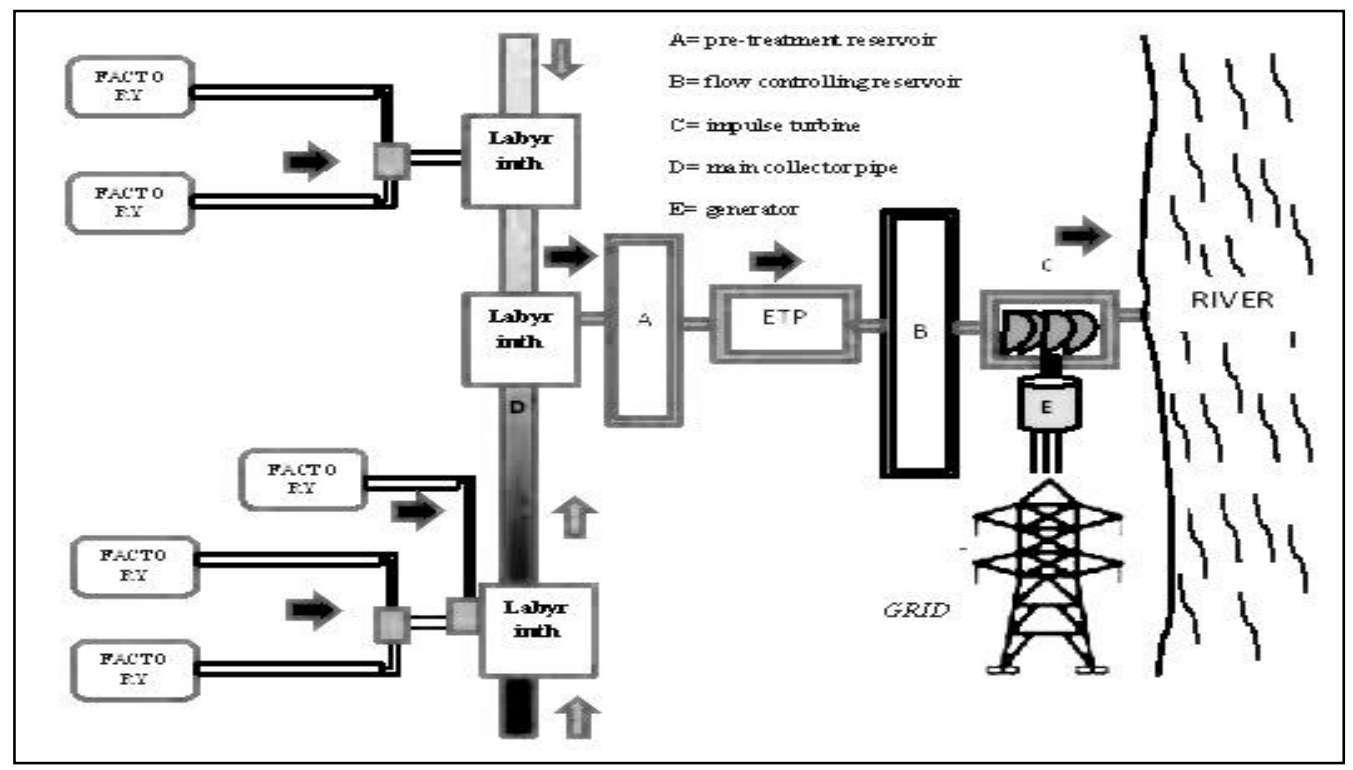




\section{Water collection \& filter Technology}

The exhaust water from the industries is collected by different flow pipes made of PVC or concrete and those converges into a main collector flow pipe. Then the mainstream heads towards a subsurface pre-treatment reservoir of dimension $100 \mathrm{~m} \times 50 \mathrm{~m} \times 15 \mathrm{~m}$. Then the water enters into ETP. ETP has three main chambers- i) dust $\&$ colloid filter ii) centrifuging portion iii) ion filter through osmosis. ETP must have the capacity to filter or purify at least $0.5 \mathrm{~m}^{3} / \mathrm{s}$ at a time. Then the purified water enters the post-treatment water reservoir having the same dimension as the previous one. This reservoir helps to maintain constant $\&$ aspect discharge rate of water.

\section{Hydro-electricity generation}

Now, the water from post-treatment reservoir having dynamic head of $15-17 \mathrm{ft}(4.6-5.2 \mathrm{~m})$ released through a PVC pipes having dia. of $9 \mathrm{ft}$ transforming a jet or nozzle of $0.5 \mathrm{ft}$.Then the water jet strikes an impulse turbine connected to a generator. The generator has a stator of permanent magnet. The torque generated on turbine tends to gyrate it and AC current is generated. The power generation formula is -

\section{Hydroelectric Power Output Estimation Formula}

Dynamic Head (m) x Flow (CMPM) x $0.18 \times$ Turbine Efficiency $(0.40)=$ Output Watts

Nozzle velocity- $v\left(m s^{-1}\right)=\frac{q\left(m^{3} s^{-1}\right)}{d_{j e t}^{2}\left(m^{2}\right)}$

The turbine peripheral velocity will be half the water jet velocity. So-

$v_{t u r b}=\frac{1}{2} \times v_{j e t}$

And turbine RPM-

$\omega_{\text {turb }}(r p m)=0.5 \times 229.2 \times \frac{v_{j e t}\left(m s^{-1}\right)}{d_{t u r b}(m)}$

Power Calculation table:

\begin{tabular}{|c|c|c|c|c|c|c|c|c|c|c|c|c|c|c|c|c|c|}
\hline \multicolumn{18}{|c|}{ power us discharge rate, nozle dia. $0.5 \mathrm{ft}$} \\
\hline $\begin{array}{l}\text { discharge } \\
\text { rate, } q \\
(\mathrm{~m} 3 / \mathrm{s})\end{array}$ & $\begin{array}{l}\text { discharge } \\
\text { rate,q } \\
\text { (USgpm) }\end{array}$ & $\begin{array}{l}\text { static } \\
\text { head (ft) }\end{array}$ & $\begin{array}{l}\text { pipe } \\
\text { (ii) }\end{array}$ & & de dia & $\mathrm{HF}(\mathrm{ft})$ & fcoef. & $\begin{array}{l}\text { Reynolds } \\
\text { no. }\end{array}$ & $\begin{array}{l}\text { pipe } \\
\text { velocity } \\
\text { (ft/s) }\end{array}$ & $\begin{array}{l}\text { nozzle } \\
\text { velocity } \\
\text { based on } \\
\text { friction } \\
\text { (Ats) }\end{array}$ & $\begin{array}{l}\text { nozle } \\
\text { velocity } \\
\text { (ftls) }\end{array}$ & $\begin{array}{l}\text { water je } \\
\text { power } \\
(\mathrm{kW})\end{array}$ & $\begin{array}{l}\text { power } \\
\text { turbine } \\
\text { wheel } \\
(\mathrm{kW})\end{array}$ & $\begin{array}{l}\text { elec. cost } \\
\text { per } \\
\text { month(\$) }\end{array}$ & $\begin{array}{l}\text { pipe } \\
\text { ength (ft) }\end{array}$ & $\begin{array}{l}\text { turbine } \\
\text { rpm }\end{array}$ & $\begin{array}{l}\text { turbine } \\
\text { dia.(ft) }\end{array}$ \\
\hline 0.5 & 132.085 & 15 & 108 & 0 & .5 & $3.8 \mathrm{E}-07$ & 0.041 & 3869.8 & 0.00463 & 31.0661 & 215.827 & 18.0212 & 8.10955 & 408.721 & 250 & 1648.92 & 15 \\
\hline 1 & 264.17 & 15 & 108 & 0 & .5 & $1.2 \mathrm{E}-06$ & 0.0333 & 7739.59 & 0.00925 & 31.0661 & 431.654 & 144.17 & 64.8764 & 3269.77 & 250 & 3297.83 & 15 \\
\hline 1.5 & 396.255 & 15 & 108 & 0 & .5 & $2.5 \mathrm{E}-06$ & 0.02977 & 11609.4 & 0.01388 & 31.0661 & 647.481 & 486.573 & 218.958 & 11035.5 & 250 & 4946.75 & 15 \\
\hline 2 & 528.34 & 15 & 108 & 0 & .5 & $4.1 \mathrm{E}-06$ & 0.0276 & 15479.2 & 0.0185 & 31.0661 & 863.308 & 1153.36 & 519.011 & 26158.1 & 250 & 6595.67 & 15 \\
\hline 2.5 & 660.425 & 15 & 108 & 0 & .5 & $6 \mathrm{E}-06$ & 0.02607 & 19349 & 0.02313 & 31.0661 & 1079.13 & 2252.65 & 1013.69 & 51090.1 & 250 & 8244.59 & 15 \\
\hline 3 & 792.51 & 15 & 108 & 0 & .5 & $8.3 \mathrm{E}-06$ & 0.02492 & 23218.8 & 0.02776 & 31.0661 & 1294.96 & 3892.58 & 1751.66 & 88283.8 & 250 & 9893.5 & 15 \\
\hline 3.5 & 924.595 & 15 & 108 & 0 & .5 & 1.1E-05 & 0.024 & 27088.6 & 0.03238 & 31.066 & 1510.79 & 6181.28 & 2781.57 & 140191 & 250 & 11542.4 & 15 \\
\hline 4 & 1056.68 & 15 & 108 & 0 & .5 & $1.4 \mathrm{E}-05$ & 0.02325 & 30958.4 & 0.03701 & 31.066 & 1726.62 & 9226.86 & 4152.09 & 209265 & 250 & 13191.3 & 15 \\
\hline 4.5 & 1188.77 & 15 & 108 & 0 & .5 & $1.7 \mathrm{E}-05$ & 0.02262 & 34828.2 & 0.04163 & 31.066 & 1942.44 & 13137.5 & 5911.86 & 297958 & 250 & 14840.3 & 15 \\
\hline 5 & 1320.85 & 15 & 108 & 0 & .5 & $2 \mathrm{E}-05$ & 0.02207 & 38698 & 0.04626 & 31.066 & 2158.27 & 18021.2 & 8109.55 & 408721 & 250 & 16489.2 & 15 \\
\hline
\end{tabular}


Power generation vs. Discharge rate Graph:

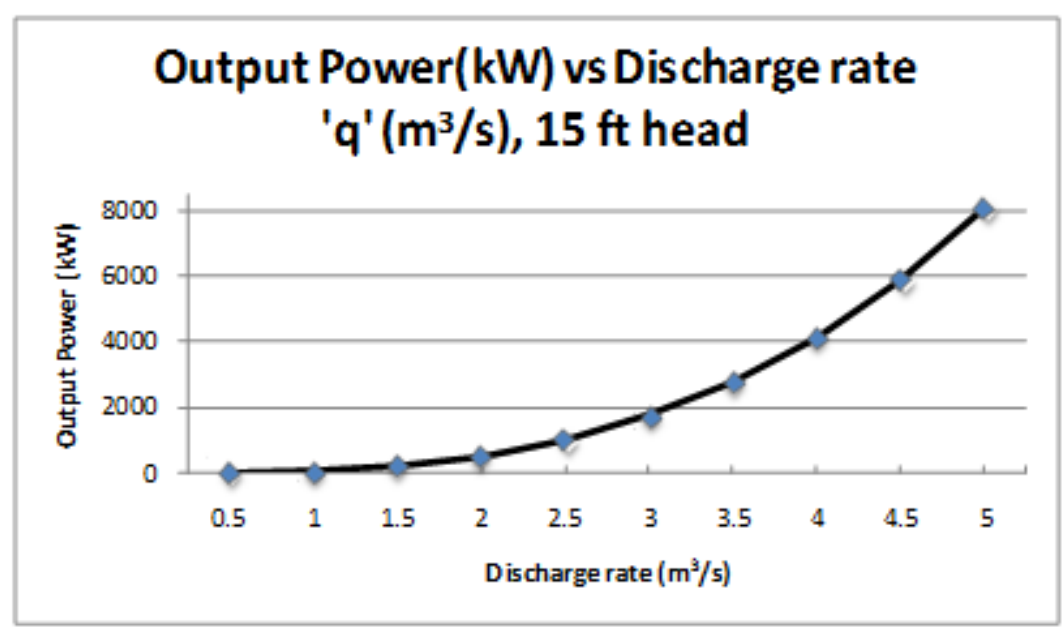

\section{Infrastructure costing:}

\section{Costing}

-The costing and installation for $5 \mathrm{~km}$ long and $10 \mathrm{ft}$. diameter concrete collector pipe having a 4 " of thickness is $=120000000$ BDT

- costing \& installation for two reservoir plant of dimension $100 \mathrm{~m} \times 50 \mathrm{~m} \times 5 \mathrm{~m}$ is $=7000000$ BDT

- ETP plant installation cost is $=227600000$ BDT

ETP plant and maintenance will be provided by textile the industry owners which are very less per head than their individual ETP running cost.

- Costing for sub-collector PVC pipes, Generator and turbine is $=20500000$ BDT

\section{Power generation costing:}

From table above, we can find that the monthly cost for $1 \mathrm{~kW}$ power generation costs $\approx 50.4 \$$

And $1 \mathrm{kWh}$ or 1 unit costs $=50.4 /(30 * 24)=0.07 \$ \approx 5.7$ BDT

Yearly saving for a $6 \mathrm{MW}$ plant $=(7.5-5.7) * 24 * 365 * 6200=97761700$ BDT

Feedback time $=$ total installation cost $/$ yearly saving $=$

$(120000000+7000000+227600000+20500000) / 97761700=3.84$ yrs.

\section{Reference:}

[1]. Bangladesh Knitwear Manufacturer \& Exporters Association, BKMEA.

[2]. Micro Hydropower, oksolar.com.

[3]. Jacques Chaurette, eng., Solving the Gravity Flow Equation for Flow Rate Using The Newton-Raphson Iteriation Technique, pp. 1-10. Feb 6, 2008.

[4]. MECH7350 Rotating Machinery, Hydraulic Turbine, chap.10, pp. 10.1-10.9, 2001. 